\title{
Welche Komponenten reflexiver beruflicher Entwicklung interessieren angehende Lehrerinnen und Lehrer? - Faktorenstruktur eines Fragebogens und erste empirische Ergebnisse
}

\section{Alois Niggli}

Der vorliegende Beitrag informiert über die Entwicklung eines Fragebogens zur Erfassung von Interessemustern zur beruflichen Reflexion im Studium von Lebrkräften. Das Verfahren umfasst drei Faktoren: (a) Interesse an der Reflexion, (b) Theoriedistanz und (c) Pragmatismus. Im Anschluss an verschiedene Pilotstudien wurde der Fragebogen an einer Stichprobe A von 210 Studierenden überprüft. Die empirischen Ergebnisse bestätigten die theoretisch postulierte Faktorenstruktur. In einer Folgeuntersuchung an einer Stichprobe (B) von 79 Personen stellte sich heraus, dass sich das Interesse an der Reflexion sowie die Theoriedistanz je nach Ausbildungsinstitution unterschiedlich entwickeln. Ferner äusserten Studierende mit Höchstnoten im berufspraktischen Examen eine signifikant geringere Theoriedistanz. Die Resultate bestätigen die Relevanz des Konzeptes.

Einleitung: Das Theorie-Praxis-Problem und die Konzeption des "Reflective Practiotioners" als Herausforderung an die Lehrerausbildung

Der Traum von der akademischen Herstellbarkeit professioneller Handlungskompetenz hat die Lehrerinnen und Lehrerbildung lange Zeit bestimmt. Inzwischen ist er wohl endgültig ausgeträumt. Die Fachwelt ist sich weitgehend einig, dass Wissenschaft und Praxis unterschiedlichen Funktionsprinzipien folgen (Terhart, 2002, S. 95; Wildt, 1996, S. 96). Konsequenterweise unterscheiden Dewe et al. (1992) auf der Grundlage ihrer Untersuchung über die Verwendung sozialwissenschaftlichen Wissens die Wissensformen des "wissenschaftlichen Wissens» und des "Handlungswissens». Auch Erkenntnisse zum Expertenwissen von Lehrerinnen und Lehrern erhärten diese Unterscheidung. Dem Expertenbzw. Handlungswissen ist nicht eine wissenschaftliche Struktur eigen. Es ist eher situations- bzw. fallorientiert und keinesfalls als allgemein zu bezeichnen (Berliner, 1992, S. 229). Daher ist zu vermuten, dass sich Lehrkräfte in Entscheidungssituationen hauptsächlich am Handlungswissen orientieren, das in ihrer 
Berufskultur entstanden ist. Gestützt wird diese Annahme durch Befragungsergebnisse, nach denen Lehrkräfte bei pädagogischen Entscheidungen zu 81.7\% überwiegend auf die eigene Berufserfahrung und nur in $7 \%$ der Fälle auf pädagogische Theorien zurückgreifen (vgl. Terhart et al. 1994, S. 196). Man kann daraus folgern, dass die beiden Wissensformen unterschiedlichen Bereichen angehören würden, die voneinander wenig lernen könnten (Dewe \& Radtke, 1991).

Eine dieser Differenzthese entgegengesetzte Position verlangt allerdings nach wie vor, dass die Pädagogik auch wissenschaftliche Hilfen zum Aufbau einer professionellen Handlungskompetenz bereitstellen müsse. Dieser integrative Ansatz wird beispielsweise im Standardkonzept (Oser, 1997) vertreten. Auch im angloamerikanischen Raum hat sich diese Perspektive längst durchsetzen können (Cochran-Smith, 2001). Standards beinhalten sowohl wissenschaftlich begründetes Wissen als auch realisierbares Können bzw. Handlungswissen. Auch Entwicklungsansätze im Rahmen der Schulentwicklung wie das "Content-Focused Coaching» (Staub, 2001; West \& Staub, 2003) setzen auf die Verbindung zwischen Praxis und fachlichem pädagogischen Wissen. In all diesen Modellen haben Lehrkräfte eine Integrationsleistung verschiedener Wissensformen zu erbringen, die Shulman als "Amalgam» bezeichnet hat (vgl. Nölle, 2002, S. 50). Erfahrene Lehrpersonen sind damit durchaus in der Lage, auf ihre Weise auch Ansprüche didaktischer Theorie zu erfüllen (Koch-Prieve, 1997). Schliesslich gilt Verwissenschaftlichung des Handelns auch als konstitutives Element einer Profession (Wildt, 1996, S. 92). Dieser Status wird für Lehrkräfte wenigstens bereichsweise eingefordert.

Folgt man der Integrationsthese, dann wird die Gestaltung komplexer (nicht linear-deduktiver) Zusammenhängen zwischen wissenschaftlichem Wissen und Handlungswissen für die Lehrerinnen- und Lehrerbildung zu einer konzeptionellen Herausforderung, die hohe Ansprüche stellt. Bei der Umsetzung dieser Forderung hat man sich vor allem vom Konzept des «reflective practitioner» (Schön, 1983, 1987, 1991; Dick 1994) entscheidend beeinflussen lassen. Diese Position besagt im Kern, dass sich das in der Ausbildung anzueignende Berufswissen einerseits in praktischen Situationen zu bewähren hat, dass es andererseits aber durch die an Begriffen und Theorien orientierte Reflexion Impulse zu seiner ständigen Entwicklung erfährt (Messner \& Reusser, 2000, S. 284). Reflexion wird damit zur Basiskonstante im beruflichen Selbstlernprozess. Ausbildungsinstitutionen sind somit in zweifacher Hinsicht gefordert:

(a) sie müssen generell eine reflexive Grundhaltung fördern;

(b) sie müssen gleichzeitig die Relationierung zwischen Theorie und Praxis herstellen können.

Die folgende Abbildung 1 veranschaulicht diese Vorstellungen. 


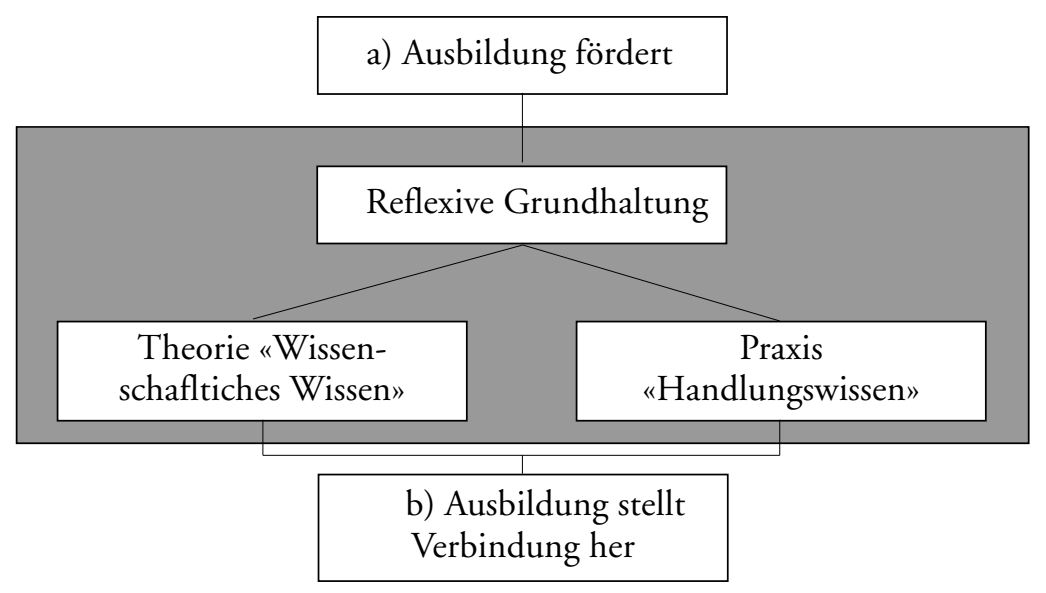

Abbildung 1: Komponenten der berufsbezogenen Reflexion in der Ausbildung von Lehrkräften

Angesichts dieser Konstellation ist es für Verantwortliche in der Ausbildung nicht unerheblich zu wissen, mit welchen Grundhaltungen zur beruflichen Entwicklung man auf Seiten der Studierenden zu rechnen hat. Wird die Integrationsthese grundsätzlich akzeptiert? Das bedeutet: Trägt die Ausbildung tatsächlich dazu bei, dass sich die Studierenden auf widerstreitende Orientierungsmuster zwischen theorie- und praxisbezogenen Interpretationswelten einlassen? Inwieweit kann die dazu notwendige reflexive Grundhaltung gefördert werden oder tendiert man eher dazu, sich in der Selbstgewissheit von Routinen und Aktionen einzurichten (Zeichner \& Liston, 1996, S. 75)? Mit dem vorliegenden Versuch wurde ein quantitatives Erhebungsinstrument entwickelt, das Hinweise liefern soll, in welchem Ausmasse sich auch Studierende an den entsprechenden Komponenten beruflicher Entwicklung orientieren. Die quantitative Datengewinnung ist ökonomisch und daher für grössere Stichproben sinnvoll. Ausbildungsverantwortliche können daraus in erster Linie Anhaltspunkte für eine gelingende Kooperation mit den Studierenden entnehmen, denn Grundhaltungen zur Ausbildung entstehen im Austausch zwischen Individuum und Ausbildungskontext. Auf längere Sicht könnte ein bewährtes Messverfahren auch prognostische Informationen liefern.

\section{Konzeption eines Fragebogens zur Erhebung reflexiver Grundhaltungen}

Selbst durch ein qualitatives Messverfahren dürfte es äusserst schwierig sein, die Vielschichtigkeit und die verschiedenen Ausprägungen des Reflexionsbegriffs zu 
erfassen (Hatton \& Smith, 1995, S. 38f.). Die vorliegende Absicht beschränkt sich deshalb darauf, das Interesse der Studierenden zu erheben, inwieweit sie sich auf den Reflexionsprozess einlassen wollen. Mit einem Fragebogen kann somit nicht der Begriff der «Reflexion» operationalisiert werden. In Übereinstimmung mit theoretischen Positionen der Interesseforschung wird Interesse vielmehr als Relation zwischen einer Person und einem Gegenstand verstanden (Krapp, 1998, S. 186). Das Interesse entwickelt sich in der Regel allmählich über vorausgegangene Erfahrungen in der Ausbildung und besitzt daher eine gewisse Stabilität. Dies ist dann der Fall, wenn die entsprechenden Erfahrungen positiv waren und die Resultate als persönlich bedeutsam eingeschätzt worden sind.

Aus dem Modell in Abbildung 1 lassen sich auf Seiten der Studierenden grundsätzlich drei unterschiedliche Interessemuster ableiten. Zum einen kann generell mehr oder weniger Interesse vorhanden sein, die eigene Berufspraxis reflexiv zu rekonstruieren. Zum anderen kann der Rekurs auf wissenschaftliches bzw. Handlungswissen jeweils variieren. Diese drei Komponenten werden auch durch theoretische Positionen zum Reflective-Practitioner-Ansatz zusätzlich untermauert (Zeichner \& Liston, 1996, S. 44 ff.). Dies ist deshalb möglich, weil sich die in Abbildung 1 dargestellten eher strukturellen Zusammenhänge auch mit korrespondierenden Dimensionen der Reflexion verknüpfen lassen. Insbesondere der Ansatz von Griffiths \& Tann (1992) ist geeignet, diese Absicht umzusetzen. Die beiden Autorinnen unterscheiden fünf Dimensionen der Reflexion. Bei den beiden Dimensionen (1) «rapid reflection» (automatische Reaktion) und (2) «repair» (überlegte Reflexion während der Handlung) handelt es sich um Prozesse, die in der Handlung selbst, «unter dem Druck der Handlung», ablaufen (vgl. Wahl, 1991). Bei den aufbauenden drei Dimensionen (3) «review» (punktuelle, wenig formalisierte Reflexion), (4) «research» (systematische, zeitlich erstreckte Reflexion) und (5) «retheorizing and research» (langzeitliche Reflexion unter Bezugnahme auf wissenschaftlich akzeptierte Informationen) handelt es sich hingegen um Aspekte der Reflexion über die Handlung («reflection on action»). Wissenschaftliche Theoriesysteme sind somit lediglich für die Dimension (5) «retheorizing and research» kennzeichnend. Es ist daher gerechtfertigt, einerseits ein generelles Interesse an Reflexion über die Praxis (on action) zu postulieren. Ferner kann eine Unterscheidung getroffen werden, ob man sich dabei mehr an praxisbezogenen (pragmatischen) Lösungen orientiert (Dimensionen 3 und 4) oder auch gewillt ist, wissenschaftliche Theoriesysteme (Dimension 5) in Betracht zu ziehen. Somit postulieren wir auf Seiten der Studierenden drei Bereiche individuellen Interesses, die mit den genannten Komponenten beruflicher Entwicklung korrespondieren (vgl. Abb. 2). Im Folgenden werden die drei Interessekomponenten näher erläutert. 


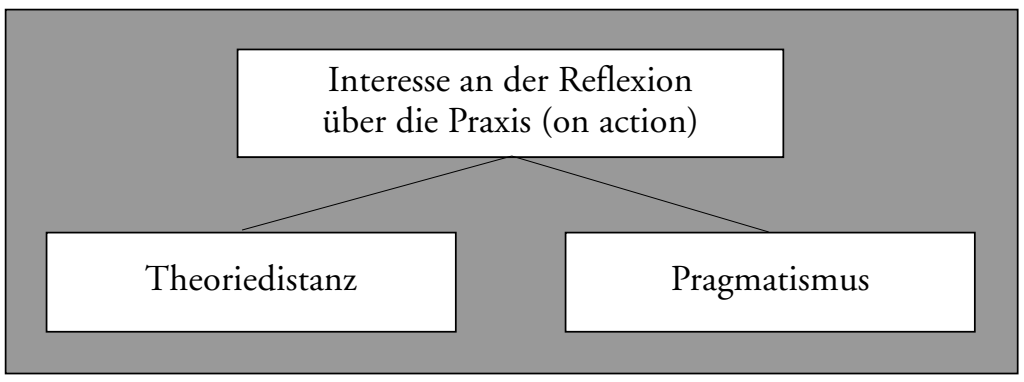

Abbildung 2: Bereiche des Interesses zu Komponenten beruflicher Entwicklung

1. Generelles Interesse an der Reflexion: Das Modell unterstellt, dass Studierende ein generelles Interesse an beruflicher Reflexion über die Handlung haben, das heisst, dass sie ihre Handlungsweise in einem kognitiven Prozess spiegeln möchten. Handeln ist ein zielgerichteter und damit ein bewusster Prozess und unterscheidet sich in dieser Beziehung von «Tun» oder «Verhalten» (Groeben, 1986). Aus der Sicht der Kontrolle des eigenen Agierens kann das reflexive Bewusstsein deshalb als Selbstüberwachungssystem bezeichnet werden (Wahl, 2002, S. 229). Reflexion ist für Handeln somit grundlegend. Sie umfasst sowohl die a-priori Entscheidungen der Planung als auch die a-posteriori Reflexion nach der Handlung. Der Reflexionsprozess kann solitär vollzogen werden. Möglicherweise ist er effizienter, wenn er im kommunikativen oder beratenden Rahmen praktiziert wird (vgl. Herzog, 1995, S. 254). Diese Merkmale der Reflexion sind zu unterscheiden von Kognitionen, die im Laufe des Tuns (reflection in action) unter Handlungsdruck unmittelbar entstehen («rapid reflection» und «repair»). Im vorliegenden Fall interessieren dagegen die im Ausbildungsprozess gestaltbaren Reflexionsanlässe (reflection on action), die von der unmittelbaren Handlungsausführung entlastet sind. Das Ausmass der Selbstüberwachung im Rahmen von «review», «research» sowie «retheorizing and research» ist deshalb abhängig vom Interesse der Beteiligten, die sich mehr oder weniger auf diese Reflexionsgelegenheiten einlassen können.

2. Theoretisches Interesse, bzw. Theoriedistanz: Gemäss den Annahmen im Reflexionsmodell sollte die Reflexion über die Handlung den Bezug zu theoretischen Positionen («Wissenschaftliches Wissen» oder «Wissen ÜBER die Praxis») einschliessen. Durch die Auseinandersetzung mit Begriffen und Theorien wird berufliches «Wissen IN der Praxis» oder «Handlungswissen» in «Wissen FÜR die Praxis» transformiert (Messner \& Reusser, 2000, S. 283). Der Rekurs auf wissenschaftlich fundierte Kommunikation ist jedoch nicht zwingend. Wissenschaftliches Wissen wird erst in der Dimension 5 «retheorizing and research» relevant. Studierende können vor allem deshalb auf Distanz zu theoriebezogenen 
Kommunikationen gehen, weil Wissenschaft von einzelnen Situationen abstrahiert und nicht den Anspruch erheben kann, direkt praxisanleitend zu sein. Es handelt sich dabei um formales, kollektives Wissen der Profession (Munby et. al., 2002, S. 881). In der Ausübung des Berufes kommt es in erster Linie aber darauf an, Regeln, die in den Routinen alltäglicher Handlungen gelten, kompetent zu handhaben. Aus Erkenntnissen der wissenspsychologischen Forschung bleibt deshalb offen, welche Relevanz das wissenschaftliche Wissen im Kontext des unterrichtspraktischen Handelns besitzt (Koch-Prieve, 2002, S. 5).

\section{Pragmatisches Interesse bzw. Pragmatismus: Pragmatismus ist konsequentes} Orientieren an der Praxis. Studierende möchten primär Konzepte oder Routinen übernehmen, die sich in der Praxis bewährt haben. Sie bevorzugen eher erfahrungsbasiertes Wissen. Ihr Interesse ist eher auf Enkulturation in das Berufsfeld ausgerichtet. Referenzpunkt für die Reflexion ist dann der praktische Kontext, gegebenenfalls auch das Modell und die subjektiven Theorien kompetenter Praktikerinnen und Praktiker. Das Interesse ist primär instrumenteller Natur. Verlässliche Informationen über Handlungserfolge sind für die Selbstwirksamkeit und Verhaltenssicherheit der angehenden Lehrerinnen und Lehrer von nicht geringer Bedeutung (Schaefers \& Koch, 2000, S. 614). Die Orientierung an der spezifischen Situation der Praxis erlaubt es, praxiswirksame (im weiteren Sinne technische) Strategien zu selektieren. Der Reflexionsprozess ist in Dimension 3 «review» wenig formalisiert («less formal», vgl. Zeichner \& Liston, 1996, S. 47). In Dimension 4 «research» wird hingegen ein systematischer, interindividueller Austausch gepflegt, der zwar eine bestimmte zeitliche Dauer beansprucht (Zeichner \& Liston, 1996, S. 47), aber ohne Bezug zu wissenschaftlichen Theoriesystemen stattfindet. Die Gefahr dabei kann darin liegen, dass im individuellen Kontext Bewährtes übernommen wird ohne auf das systematisierte wissenschaftliche Wissen zurückzugreifen, das man nicht selbst erfahren hat. Das Interesse an der theoriebezogenen Reflexion und eher pragmatisch orientierte Zugänge sind im Modell von Griffiths \& Tann (1992) jedoch unterschiedliche Dimensionen einer reflektierten Berufsauffassung, die sich gegenseitig ergänzen.

\section{Methode}

\section{I t emsammlung}

Auf dem Hintergrund der im obigen Rahmenkonzept beschriebenen Interessekomponenten war eine Itemsammlung zu erstellen. Verfahren mit vergleichbarem Gültigkeitsanspruch existieren nicht. Infolgedessen wurden Items aufgrund von Befragungen von Studierenden und aufgrund von Theorie-Praxis-Bemerkungen in schriftlichen Arbeiten der Studierenden formuliert. Diese Itemsammlung wurde mit Studierenden diskutiert und überarbeitet. Anschliessend wurde die Gültigkeit mit dem Rahmenkonzept durch Experten geprüft. Schliesslich 
konnte den Studierenden eine Sammlung von 38 Fragen zur Beantwortung vorgelegt werden. Die Items waren auf einer sechsstufigen Likertskala einzuschätzen, und zwar zwischen den Polen «lehne gänzlich $a b$ » und «stimme voll zu».

\section{Stichprobe und Durchführung}

Stichprobe A: Eine erste Stichprobe wurde zur Entwicklung des Fragebogens herangezogen. Der Fragebogen wurde im Studienjahr 2001 insgesamt 210 Studierenden zur Beantwortung vorgelegt. 85 (40\%) Personen waren männlichen, 123 (60\%) weiblichen Geschlechts (2 Personen ohne Angabe des Geschlechts). Stichprobe A umfasste 186 Studierende der Sekundarstufe I und 24 Studierende eines Lehrerinnen- und Lehrerseminars.

Zusätzliche Angaben zu den Studierenden der Sekundarstufe I: Die 186 Studierenden stammten aus drei universitären Ausbildungsinstitutionen für Lehrerinnen und Lehrer der Sekundarstufe I. Die Lehrgänge wiesen eine ähnliche Ausbildungsstruktur auf. In den ersten sechs Semestern wurden die verschiedenen Ausbildungsbereiche integriert angeboten. Die letzten beiden Semester 7 und 8 waren ausschliesslich für die erziehungswissenschaftliche und berufspraktische Ausbildung reserviert. 93 Personen dieser Studierenden bilden eine erweiterte Standardgruppe, die sich durch ein auf den Unterricht abgestimmtes Theoriecurriculum auszeichnet, das mit praktischen Erprobungen in Praxiseinsätzen verbunden war. Diese Erprobungen wurden vor dem Hintergrund von Theorie und Ausführbarkeit reflektiert und zu verbessern versucht. Damit wurde die Theorie - sicher zunächst nur annäherungsweise - auf professionelle Standards des Lehrerberufs hin orientiert. Die Studierenden der Standardgruppen stammen aus einem Lehrgang der Universität Freiburg (Schweiz). Da es sich um eine Querschnittuntersuchung handelt, wird in Tabelle 1 die Verteilung auf die verschiedenen Studiensemester angegeben.

Tabelle 1: Verteilung auf die verschiedenen Studiensemester

\begin{tabular}{|c|c|c|c|c|c|c|}
\hline & Semester 1 & Semester 3 & Semester 5 & Semester 7 & Semester 8 & Total \\
\hline Standardgruppe & 16 & 7 & 33 & 37 & $\begin{array}{c}\text { s. Stichprobe } \\
\text { B }\end{array}$ & 93 \\
\hline Kontrollgruppe & & 27 & 21 & 1 & 44 & 93 \\
\hline Total & $16(9 \%)$ & $34(18 \%)$ & $54(29 \%)$ & $38(20 \%)$ & $44(24 \%)$ & 186 \\
\hline
\end{tabular}

70 (38\%) Studierende der Sekundarstufe I hatten die mathematisch-naturwissenschaftliche Studienrichtung gewählt. 116 (62\%) Studierende belegten die sprachlich-historische Fachausbildung.

Zusätzliche Angaben zu den Studierenden eines Lehrerinnen- und Lehrerseminars für die Primarstufe: Die 24 Studierenden (ca. 11\% der Gesamtgruppe) befanden sich im letzten Ausbildungsjahr (10. Semester). Es handelt sich dabei um 21 Frauen und 3 Männer. 
Stichprobe B: Eine zusätzliche Stichprobe wurde verwendet, um Zusammenhänge zwischen den im Fragebogen gemessenen Interessen und dem didaktischem Können zu untersuchen. Die Erhebung wurde in den Jahren 2002 und 2003 durchgeführt. Es handelte sich ausschliesslich um Studierende der Institution mit der oben beschriebenen standardorientierten Ausbildungsgang. Sie umfasste 23 (30\%) Männer und 50 (70\%) Frauen. 57 (78\%) Kandidatinnen und Kandidaten erwarben ein Lehrdiplom sprachlich historischer Richtung, 16 (12\%) Personen hatten Mathematik und Naturwissenschaften belegt. Von 6 Personen waren keine Angaben zu erhalten. Die Probandinnen und Probanden beantworteten den Fragebogen vor der Durchführung ihrer lehrpraktischen Prüfung, die frühestens am Ende des 8. Semesters abgelegt werden kann.

\section{Datenanalysen}

Überprüfung des Fragebogens: Die Itemkonstruktion wurde aufgrund eines theoretischen Rahmenmodells vorgenommen. Die Untersuchung der Faktorenstruktur ist deshalb unter dem Aspekt der Skalenkonstruktion zu sehen. Es ging um die Frage, ob eindeutige Itemzuordnungen für die vorgesehenen Skalen gewährleistet sind. Die endgültige Zusammenstellung der Items wurde durch die üblichen Item- und Skalenanalysen der klassischen Testtheorie überprüft. Anhand der Interkorrelationen der Skalen wurde geprüft, inwieweit die konstruierten Skalen unabhängig voneinander sind. Als weiterer Aspekt der Validität wurden Korrelationen zu zwei geeigneten FSI-items zum Studieninteresse (Schiefele et al., 1993) gerechnet.

Vergleichsuntersuchungen: Im Rahmen des Reflexionsmodells zur Ausbildung von Lehrkräften sind diagnostische Informationen über die Ausprägung der drei postulierten Grundhaltungen quasi ein Ziel an sich. Gleichzeitig geht damit automatisch die Frage einher, welchen Bedingungen sie unterliegen. Als unabhängige Variable interessierten Angaben zur Person der Befragten (Geschlecht und Studienwahl), ferner die erhobenen Merkmale im Ausbildungsgang (Semesterzahl sowie die unterschiedliche Standardorientierung). In einer zusätzlichen Fragestellung wurde geprüft, ob ein Einfluss der drei Grundhaltungen auf das praktische Können der Studierenden in der Lehrprobe, die am Ende der Studien stattfindet, nachgewiesen werden kann. Interesse an der Reflexion, Theoriedistanz und Pragmatismus wurden somit auch in ihrer Bedeutung als unabhängige Variable untersucht. 


\section{überprüfung des Fragebogens zur Erhebung reflexiver Interessen}

\section{Faktorielle Struktur}

Das theoretische Rahmenkonzept wurde durch eine Hauptkomponentenanalyse mit anschliessender Varimaxrotation überprüft. Dabei stand die Frage im Vordergrund, ob eindeutige Itemzuordnungen für die drei vorgesehenen Skalen gewährleistet sind. Es handelt sich somit um ein strukturprüfendes empirisches Klassifikationsverfahren.

Zunächst wurde kontrolliert, ob die Korrelationsmatrix überhaupt dazu geeignet war, um mit ihr eine Faktorenanalyse durchzuführen (Programm SPSSfMac). Der MSA Wert (Measure of Sampling Adequacy) betrug .71, was als «ziemlich gut» gelten kann. Mit der Faktorenanalyse wurde anschliessend geprüft, ob der Itempool durch drei Komponenten erster Ordnung in der vorhergesagten Weise strukturiert werden kann. Der Eigenwertverlauf wies 13 Eigenwerte mit einem Wert grösser 1 auf $(\mathrm{E} 1=5.14$, E2 = 3.63, E3 = 1.9, E4 = 1,74, $\mathrm{E} 5=1,57, \ldots \mathrm{E} 13=1.05)$. Der Scree-Test legte allerdings nur die Extraktion von 3 Faktoren nahe. Die Differenzen der Eigenwerte E1 bis E3 sind bedeutend, ab E3 unterscheiden sie sich nur mehr unwesentlich. Zeichnet man eine Screetestkurve, dann bildet die Kurve nach dem dritten Wert einen Knick. Dies ist ein Hinweis dafür, dass eine Dreifaktorenlösung angemessen ist. Insgesamt konnten 16 Items selektioniert werden, die nur auf einem dieser drei Faktoren laden. Die theoretischen Vorannahmen sind somit numerisch bestätigt worden. Mit diesen ausgewählten 16 Items wurde eine zweite Faktorenanalyse gerechnet. Die Ergebnisse dieser 3-Komponentenlösung, die insgesamt 43\% der Gesamtvarianz erklärt, sind in Tabelle 2 dargestellt.

Tabelle 2: Itemkennwerte der drei Faktoren (Faktorladungen, Arithmetisches Mittel und Standardabweichungen)

\begin{tabular}{|c|c|c|c|c|c|}
\hline Faktor I: Theoriedistanz & $\mathrm{F}_{1}$ & $\mathrm{~F}_{2}$ & $\mathrm{~F}_{3}$ & $\mathrm{AM}$ & SD \\
\hline $\begin{array}{l}\text { 15. Wenn ich ehrlich sein soll, sind mir theoretische } \\
\text { Vorstellungen über die Erziehung manchmal eher } \\
\text { gleichgültig. }\end{array}$ & .71 & .19 & .13 & 3.69 & 1.37 \\
\hline $\begin{array}{l}\text { 11. Die meisten Resultate von erziehungswissenschaftli- } \\
\text { chen Studien sind für die Praxis mehr oder weniger be- } \\
\text { deutungslos. }\end{array}$ & .68 & .11 & .03 & 3.17 & 1.16 \\
\hline $\begin{array}{l}\text { 3. Im Unterrichtsalltag kommt man meistens sehr gut } \\
\text { auch ohne Theorien aus. }\end{array}$ & .67 & .19 & .17 & 3.51 & 1.36 \\
\hline $\begin{array}{l}\text { 16. Ich habe selbst didaktische Bücher gekauft, die mir } \\
\text { Impulse für meinen Unterricht geben können.* }\end{array}$ & .66 & .14 & .03 & 3.16 & 1.63 \\
\hline 6. Theorien über Unterricht bringen meist nicht viel. & .62 & .27 & .11 & 3.32 & 1.21 \\
\hline $\begin{array}{l}\text { 8. Lehrerinnen und Lehrer sollten beim praktischen } \\
\text { Handeln unbedingt auch wissenschaftliche Kenntnisse } \\
\text { berücksichtigen.* }\end{array}$ & .56 & .02 & .07 & 4.24 & 1.05 \\
\hline
\end{tabular}




\begin{tabular}{|c|c|c|c|c|c|}
\hline Faktor II: Pragmatismus & $\mathrm{F}_{1}$ & $\mathrm{~F}_{2}$ & $\mathrm{~F}_{3}$ & $\mathrm{AM}$ & SD \\
\hline $\begin{array}{l}\text { 12. Ich orientiere mich am liebsten an Konzepten von } \\
\text { Kolleginnen und Kollegen oder von Praxislehrkräften. }\end{array}$ & .24 & .70 & .06 & 3.46 & 1.06 \\
\hline $\begin{array}{l}\text { 9. Nur Lehrmittel, die auch Kopiervorlagen für den } \\
\text { Unterricht anbieten, bringen wirklich etwas. }\end{array}$ & .05 & .69 & .03 & 3.08 & 1.40 \\
\hline $\begin{array}{l}\text { 5. Den grössten Nutzen für meinen Unterricht bringen } \\
\text { mir Tipps von Kolleginnen und Kollegen. }\end{array}$ & .16 & .61 & .29 & 4.28 & 1.01 \\
\hline $\begin{array}{l}\text { 14. Unterrichten ist in erster Linie ein Handwerk, das } \\
\text { man von erfahrenen Lehrkräften übernimmt. }\end{array}$ & .12 & .56 & .13 & 3.16 & 1.16 \\
\hline $\begin{array}{l}\text { 2. Im Unterricht wende ich nur Prinzipien an, die sich } \\
\text { in der Praxis auch bewährt haben. }\end{array}$ & .05 & .53 & .15 & 3.46 & 1.29 \\
\hline Faktor III: Interesse an Reflexion & $\mathrm{F}_{1}$ & $\mathrm{~F}_{2}$ & $\mathrm{~F}_{3}$ & $\mathrm{AM}$ & SD \\
\hline $\begin{array}{l}\text { 10. Nur wenn man sein Handeln im Unterricht immer } \\
\text { wieder in Frage stellen kann, wird man eine gute Leh- } \\
\text { rerin oder ein guter Lehrer. }\end{array}$ & .08 & .08 & .66 & 5.10 & .90 \\
\hline $\begin{array}{l}\text { 13. Nachüberlegungen zum Unterricht sind genau so } \\
\text { wichtig wie die Unterrichtsplanung. }\end{array}$ & .20 & .18 & .63 & 5.04 & .84 \\
\hline $\begin{array}{l}\text { 1. Etwas vom wichtigsten im Beruf als Lehrerin oder } \\
\text { als Lehrer ist, sich jeweils immer genau zu überlegen, } \\
\text { wie man vorgehen will. }\end{array}$ & .03 & .13 & .59 & 4.87 & .93 \\
\hline $\begin{array}{l}\text { 4. Wenn man im Unterricht Probleme hat, dann } \\
\text { bespricht man sie am besten mit Kolleginnen und } \\
\text { Kollegen. }\end{array}$ & .06 & .19 & .49 & 5.19 & .80 \\
\hline $\begin{array}{l}\text { 7. Nur wenn man auch Denkanstösse von aussen ernst } \\
\text { nimmt, lässt sich der eigene Unterricht wirksam über- } \\
\text { prüfen und verbessern. }\end{array}$ & .16 & .14 & .53 & 4.92 & .82 \\
\hline
\end{tabular}

* Item 16 und 8: negative Polung

Die inhaltlichen Zuordnungen der Items zu den drei Faktoren lassen sich wie folgt zusammenfassen.

Faktor I: Theoriedistanz: Distanz zu theoretischen Vorstellungen wird nicht ausschliesslich nur im schulischen Kontext, sondern generell gegenüber erzieherischem Handeln geäussert (höchste Ladung Item Nr. 15). Diese Orientierung zeigt sich auch auf der Verhaltensebene, wenn etwa angegeben wird, dass man wenige didaktische Bücher kaufe (Item 16).

Faktor II: Pragmatismus: Studierende wollen auch durch Beobachtung und Nachahmung lernen. Man orientiert sich dabei an Konzepten von erfahrenen Lehrkräften (höchste Ladung Item Nr. 12), ist aber auch bereit, Tipps von Kolleginnen und Kollegen zu beachten. Auch von Lehrmitteln verlangt man, dass sie konkrete Hilfen (Kopiervorlagen, Item 9) enthalten. Für die Items, die auf dem Pragmatismusfaktor laden, ist charakteristisch, dass sie in der Mehrzahl eine eher ökonomische Komponente enthalten (Tipps und Bewährtes übernehmen, 
Kopiervorlagen nutzen usw.). Insgesamt möchte man von Erfahrungen, die andere gemacht haben, profitieren können.

Faktor III: Interesse an der Reflexion: Dieser Faktor ist inhaltlich heterogener strukturiert als die beiden ersten Faktoren. Er umfasst sowohl planerische apriori Vorüberlegungen (Item Nr. 1), als auch die a-posteriori Reflexion über das Handeln (Item Nr. 13) . Generell ist man bereit, sein Handeln immer wieder in Frage zu stellen (höchste Ladung Item Nr. 10) und auch Anstösse von aussen anzunehmen. Dazu gehört, dass man bereit ist, Probleme im kommunikativen Rahmen zu besprechen (Item 4).

Die drei Faktoren entsprechen somit auch inhaltlich den theoretischen Vorannahmen.

\section{Skalenbi ldung}

Tabelle 2 enthält die Skalenbezeichnungen, Verteilungsstatistiken und die Reliabilitätskoeffizienten (Cronbachs Alpha). Die Skala Theoriedistanz ist inhaltlich relativ homogen $($ Alpha $=.74)$. Die jeweiligen Items sind sich inhaltlich ähnlich. Weil sie Identisches messen, ist ihre innere Konsistenz deutlich höher als diejenige von Faktor III, der sich aus einem Bündel verschiedenster Reflexionsmöglichkeiten zusammensetzt.

\section{Tabelle 3: Verteilungswerte und interne Konsistenzen der Skalen}

\begin{tabular}{|l|c|c|c|c|c|c|}
\hline Skala & AM & Median & SD & Min. & Max. & Alpha \\
\hline Theoriedistanz (6 Items) & 20.33 & 20.00 & 5.1 & 9.00 & 34.02 & .75 \\
Pragmatismus (5 Items) & 17.49 & 18.00 & 3.78 & 5.00 & 26.00 & .63 \\
Reflexion (5 Items) & 25.12 & 25.00 & 2.62 & 18.00 & 30.00 & .57 \\
\hline
\end{tabular}

Die Skala Theoriedistanz streut relativ auffällig. Der Mittelwert liegt im mittleren Skalenbereich (gerundete durchschnittliche Beantwortung auf der sechsstufigen Likert-Skala: 20.33 / 6 Items = 3.38). Überaus hohe Zustimmung finden generell die Reflexionsitems. Infolgedessen ist die Streuung bei dieser Skala gering. Im mittleren Intervallbereich liegen die Werte für die Pragmatismus-Skala. Die Pragmatismusorientierung ist jedoch deutlich weniger auffällig als das Interesse an Reflexion. Die Item- und die Skalenanalysen wurden am gleichen Datenmaterial durchgeführt. Bei den berichteten Kennwerten handelt es sich somit um Parameter einer Basisversion. Wünschenswert wäre eine Kreuzvalidierung an einer neuen Stichprobe.

Analysen zur Validität

Die Korrelationsmatrix der Skalen ist durch niedrige Werte gekennzeichnet. Kein Zusammenhang besteht zwischen Pragmatismus und Reflexion. Die bei- 
den Skalen variieren voneinander unabhängig. Die niedrigen Korrelationen zwischen Theoriedistanz sowie Pragmatismus und Reflexion sind ebenfalls ein Indiz für die diskriminante Validität der Skalen.

Tabelle 4: Interkorrelationen der Skalen

\begin{tabular}{|l|c|c|c|}
\hline & Theoriedistanz & Pragmatismus & Reflexion \\
\hline Theoriedistanz & 1 & .27 & -.28 \\
Pragmatismus & & 1 & -.09 \\
Reflexion & & & 1 \\
\hline
\end{tabular}

Einsichtig ist der negative Zusammenhang zwischen Theoriedistanz und dem Interesse an der Reflexion über die Praxis, weil reflexives Handeln wohl immer theoretisierende Elemente (wissenschaftliche oder subjektive Theorien) einschliesst. Insgesamt stützen die drei niedrigen Korrelationen die Ausgangshypothese, dass eine Trennung der drei Skalen auf dispositioneller Ebene möglich ist.

Zur Überprüfung der Konstruktvalidität wurden zwei inhaltlich geeignete Items des FSI (Fragebogen zum Studieninteresse) von Schiefele et al. (1993) berufsbezogen umformuliert und mit den drei reflexiven Interesseskalen korreliert. Die beiden Items lauten wie folgt: «Über Unterricht zu reden macht mir selten Spass.» und «Ich rede lieber über meine Hobbies als über Unterricht». Der Begriff «Studienfach» im FSI wurde durch «Unterricht» ersetzt. Die negative Korrelation zwischen den beiden adaptierten FSI-Items und der Skala Theoriedistanz beträgt -.43. Je geringer das Interesse am Unterricht generell ist, desto ausgeprägter ist die Theoriedistanz. Mit der Skala Reflexion korrelieren die beiden Items hingegen positiv mit .36. Eine Nullkorrelation ergab sich zum Pragmatismus.

\section{Ergebnisse der Vergleichsuntersuchungen}

\section{Vergleich zwischen Bedingungsmerkmalen der Stichprobe sowie Theoriedistanz, Pragmatismus und Interesse an Reflexion als abhängige Variablen}

Eine Bedingungsanalyse kann sich im momentanen Stadium vorerst auf relativ einfache Fragestellungen konzentrieren. Im Vordergrund stand die Abhängigkeit von Geschlecht und Studienrichtung. Es handelt sich dabei um personenbezogene Bedingungsvariablen. Die erweiterte Standardorientierung und die Semesterzahl betreffen Merkmale der Ausbildungsstruktur. Tabelle 5 vermittelt eine Übersicht über die signifikanten Zusammenhänge. Die Analysen wurden an Stichprobe A vorgenommen. 
Tabelle 5: Überblick über Theoriedistanz, Pragmatismus und Interesse an der Reflexion als abhängigen Variablen

\begin{tabular}{|l|c|c|c|}
\hline & Theoriedistanz & Pragmatismus & Reflexion \\
\hline Studienrichtung & -- & -- & -- \\
Geschlecht & -- & -- & sig. \\
Semesterzahl & sig. & -- & sig. \\
Standard & sig. & -- & sig. \\
\hline
\end{tabular}

Von den vier Bedingungsfaktoren hat sich die gewählte Studienrichtung (Ausbildung zur Lehrkraft für die Sekundarstufe I: Mathematik und Naturwissenschaften bzw. Sprachlich-historische Richtung; Primarlehrkraft) als unbedeutend erwiesen. Im Weiteren scheint keine der genannten vier Bedingungsvariablen auf beruflichen Pragmatismus einen Einfluss zu haben. Signifikante Differenzen beschränken sich infolgedessen auf die Theoriedistanz sowie das Interesse an der Reflexion. Bedingt sind sie durch das Geschlecht, die Semesterzahl und vor allem durch die erweiterte Standardorientierung. Im Folgenden werden die signifikanten Ergebnisse im Einzelnen erläutert.

a) Vergleich zwischen dem Geschlecht und dem Interesse an Reflexion: Das Geschlecht scheint das generelle Interesse an Reflexion zu beeinflussen. Dieses scheint bei Frauen etwas ausgeprägter zu sein als bei Männern (Frauen AM = $5.11, s=.53$; Männer $A M=4.90, s=50)$. Die Unterschiede sind signifikant $(F$ $=7.82$, df 206, $\mathrm{p}=.005)$. Allerdings bewegen sich die Mittelwerte für beide Geschlechter auf einem sehr hohen Niveau. Diese Ergebnisse beruhen auf Stichprobe $A$.

b) Vergleich zwischen der Anzahl Studiensemestern und den drei Interesseskalen: Im Hinblick auf die Gestaltung von Studienplänen ist auch eine allfällige Variation im zeitlichen Verlauf einer Ausbildung prüfenswert. Die Dauer der Ausbildung scheint die drei Interessekomponenten nur punktuell zu beeinflussen. In Abbildung 3 sind die Verläufe für die Standardgruppe dargestellt. Die Beschränkung auf die Sandardgruppe verhindert Verzerrungen durch unterschiedliche Studienpläne. Die Interpretationen der Ergebnisse lassen Hinweise zum Studienverlauf zu.

In einem Querschnittvergleich wurden die Werte von Studierenden im ersten, fünften und siebten Semester, sowie kurz vor Studienabschluss, in der Regel Ende des achten Semesters, untersucht. Die Theoriedistanz verharrte bis zum siebten Semester auf konstantem Niveau. Hingegen konnte im letzten Ausbildungsjahr ein signifikanter Rückgang beobachtet werden (Einfaktorielle Varianzanalyse: $\mathrm{F}=5.031, \mathrm{df}=171, \mathrm{p}=.001)$. Bei den Studierenden des siebten Semesters handelt es sich um die Standardgruppe von Stichprobe A. Der Rückgang 
im achten Semester betraf die Studierenden der Stichprobe B, die derselben Ausbildungsinstitution angehören.

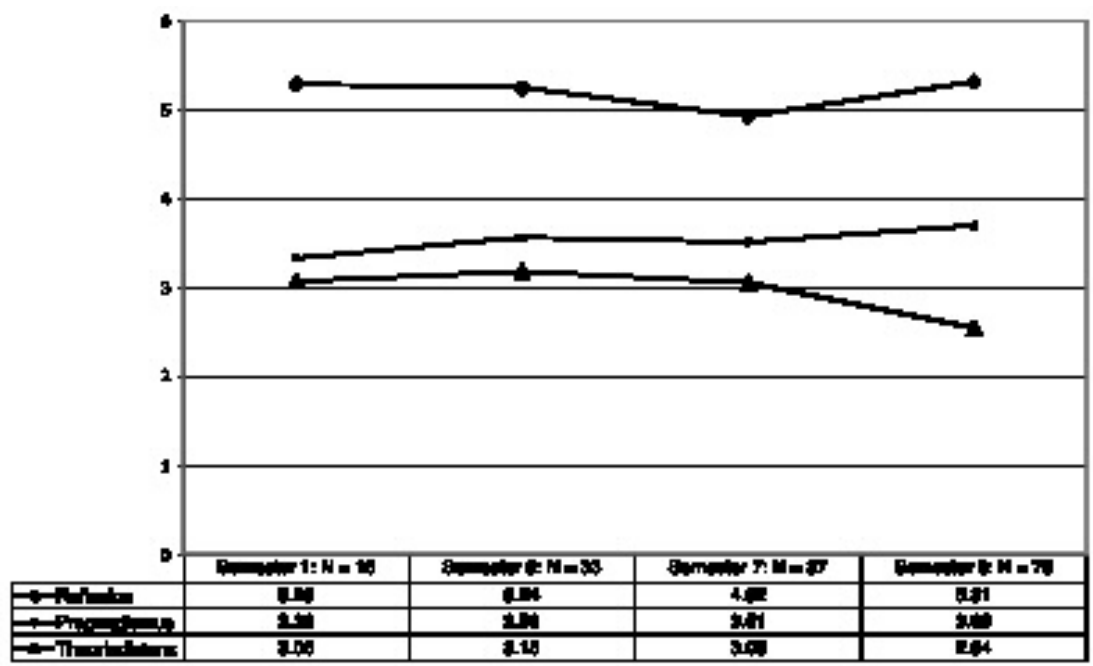

Abbildung 3: Querschnittvergleich von Interesse an Reflexion, Pragmatismus und Theoriedistanz im Studienverlauf der Standardgruppe SEK I (Stichproben A und B)

Dazu ist zu bemerken, dass die standardorientierte Ausbildung in den letzten beiden Ausbildungssemestern aus organisatorischen Gründen intensiviert werden kann. Die wahrnehmbare Differenz zum üblichen akademischen Studienbetrieb ist in diesem Schlussjahr somit am auffälligsten. In den ersten sechs Semestern wird dagegen eine integrierte Ausbildung absolviert, wo die Fachstudien den grössten Ausbildungsanteil beanspruchen und standardorientierte Module nur punktuell im didaktischen Bereich angeboten werden können. In einem vergleichbaren Kontext kann die Abnahme des Interesses an der Reflexion zu Beginn des siebten Semesters gesehen werden $(F=3.921, \mathrm{df}=169, \mathrm{p}=.005)$. Während dem ersten und während dem fünften Semester sind die Praxiseinsätze des Grundstudiums zu leisten. Ab dem sechsten bis zu Beginn des siebten Semesters sind die Studierenden in erster Linie mit Fachprüfungen konfrontiert. Die Gelegenheiten zur Praxisreflexion sind infolgedessen limitiert. Keine signifikanten Unterschiede waren hingegen beim Studienverlauf der Kontrollgruppe feststellbar. Die Messzeitpunkte waren hier im dritten, fünften und während dem achten Semester angesetzt. (In diese Analyse wurden wiederum nur die Studierenden des Sekundarlehramtes einbezogen. Die Substichprobe der Primarlehrkräfte umfasste lediglich einen einzigen Jahrgang.) 
c) Vergleich zwischen unterschiedlichen Ausbildungsgängen sowie Theoriedistanz und dem Interesse an der Reflexion: Die in Abbildung 1 formulierten Modellvorstellungen weisen der Ausbildungsinstitution eine vergleichsweise zentrale Bedeutung zu, wenn es darum geht, den Reflexionsprozess zu fördern bzw. wissenschaftliches und Handlungswissen zu verbinden. Diese Annahmen haben sich empirisch bestätigt. Sowohl Theoriedistanz als auch das Interesse an der Reflexion können zwischen Ausbildungsinstitutionen variieren. Hoch signifikante Unterschiede wurden zwischen einer Kontrollgruppe und Studierenden beobachtet, die ein erweitertes an didaktischen Ausbildungsstandards orientiertes Ausbildungskonzept (vgl. Oser, 1997) durchlaufen, in dem theoretische und praktische Komponenten reflexiv aufeinander bezogen werden. Abb. 4. veranschaulicht die Ergebnisse.

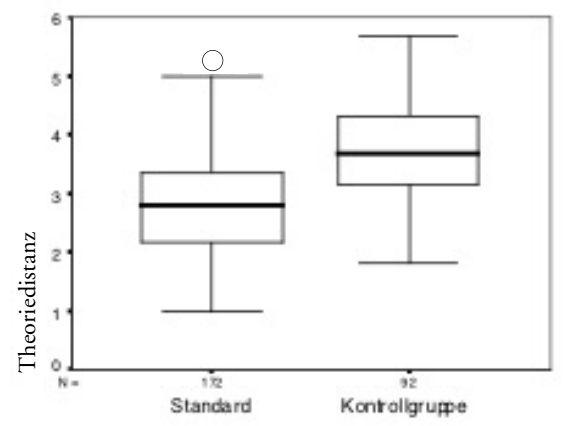

Standardgruppe: $\quad$ Median $=2.8 ; \mathrm{AM}=2.8, \mathrm{~s}=.84$ Kontrollgruppe: $\quad$ Median $=3.6 ; \mathrm{AM}=3.7, \mathrm{~s}=.82$ $\mathrm{F}=65.326, \mathrm{df}=1 / 262, \mathrm{p}=.000$

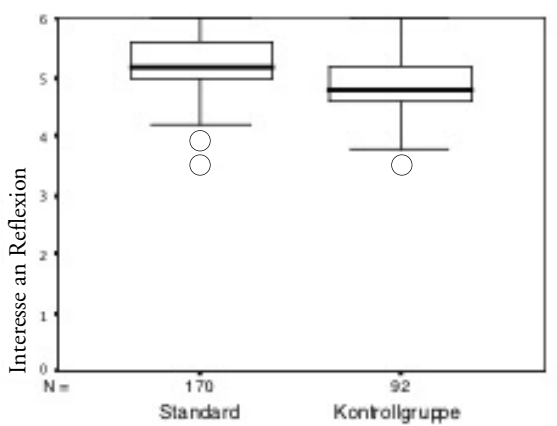

Standardgruppe: $\quad$ Median $=5.2 ; \mathrm{AM}=5.2, \mathrm{~s}=.52$ Kontrollgruppe: $\quad$ Median $=4.8 ; \mathrm{AM}=4.9, \mathrm{~s}=.48$ $\mathrm{F}=21.762, \mathrm{df}=1 / 260, \mathrm{p}=.000$

Abbildung 4: Zusammenhänge zwischen Ausbildungsinstitution, Theoriedistanz und Interesse an der Reflexion (Studierende der Sekundarstufe I: Stichproben A und B)

Studentinnen und Studenten der erweiterten Standardgruppe äussern deutlich geringere Theoriedistanz als Studierende einer vergleichbaren Kontrollgruppe (Differenz der Mediane 0.8). Ebenfalls signifikant ist die Differenz zum Interesse an der Reflexion. Der Abstand der beiden Mediane reduziert sich in diesem Fall jedoch auf den Wert von 0.4.

\section{Vergleich zwischen Theoriedistanz, Pragmatismus, dem Interesse an der Reflexion als unabhängige Variablen so- wie den Abschlussnoten in der Lehrprobe}

Als relevant erachtete Komponenten beruflicher Entwicklung sollten das Können der Studierenden nicht unbeeinflusst lassen. Zu diesem Zwecke wurde untersucht, inwieweit die drei Interesseskalen mit den Leistungen in den Abschlusslehrproben der Kandidatinnen und Kandidaten zusammenhängen 
(siehe Stichprobe B). Während sich für das Interesse an der Reflexion und auch zu pragmatischen Vorstellungen keine Zusammenhänge einstellten, erwies sich die Variable "Theoriedistanz» gewissermassen als Schlüsselfaktor. Eine signifikante Differenz konnte festgestellt werden zwischen Studierenden, deren Lehrprobe als exzellent (Note 6) bewertet worden ist und Studierenden die sehr gute bis genügende Leistungen erbracht hatten. Die Daten stützen sich auf zwei unterschiedliche Abschlussjahrgänge. Die Urteile stammen von vier Experten allgemeindidaktischer Richtung, die jeweils zusammen mit den entsprechenden Fachdidaktikdozierenden eine Note setzten. Die Leistungen wurden wie folgt benotet: Exzellent (Note 6), ausgezeichnet (Note 5,5), solide (Note 5). Sechs Studierende erzielten unterdurchschnittliche Leistungen, eine Person war knapp genügend, eine Person ungenügend. Die Kandidatinnen und Kandidaten mit unterdurchschnittlichen Ergebnissen konnten aufgrund des zu kleinen $\mathrm{N}$ nicht in die untenstehende Abbildung 5 aufgenommen werden. Sie figurieren jedoch in der für die Berechnung der Signifikanz verwendeten Gesamtstichprobe. Gerechnet wurde ein t-Test mit dem «Cutpoint» zwischen der Note 5.5 (ausgezeichnet) und 6 (exzellent). Danach äussern 22 Studierende mit Note 6 (exzellent) signifikant geringere Theoriedistanz als die übrigen 57 Studentinnen und Studenten mit dem Notenspektrum von ausgezeichnet bis unterdurchschnittlich (Studierende mit Note 6: $\mathrm{AM}=2.24, \mathrm{~s}=.559 /$ übrige Studierende: $\mathrm{AM}=2.66$, $\mathrm{s}=.819) ;(\mathrm{t}=-2.075, \mathrm{df}=77, \mathrm{p}=0.04)$. Das Kriterium homogener Varianzen war erfüllt (Test von Levine: $\mathrm{F}=6.554, \mathrm{p}=0.01$ ).

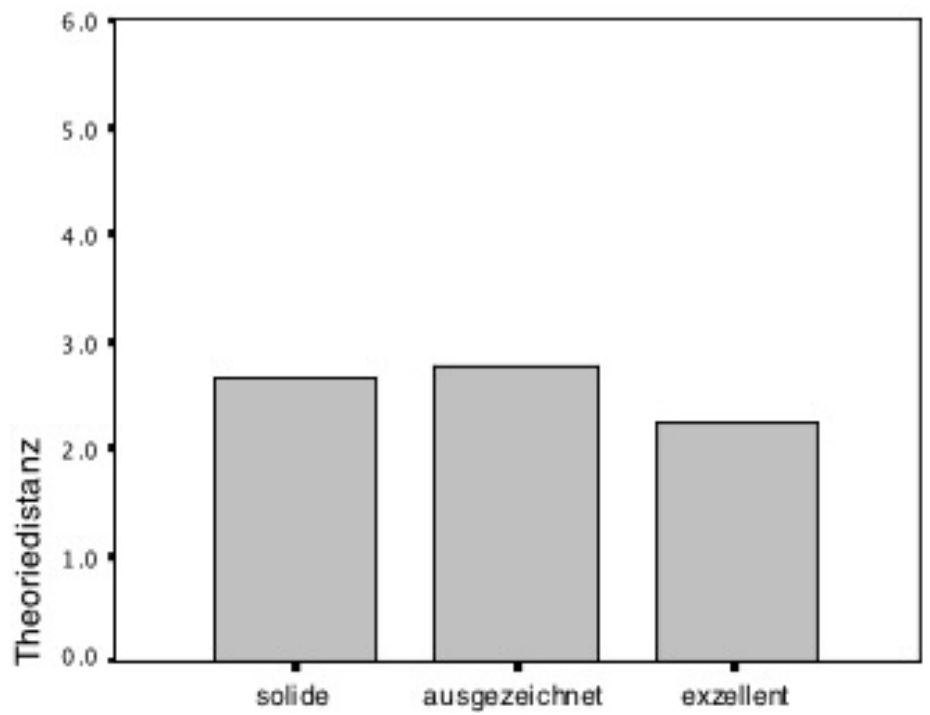

Abbildung 5: Theoriedistanz von Kandidatinnen und Kandidaten mit unterschiedlichen Abschlussnoten in der Lehrprobe (exzellent: $N=22, A M=2.24, s=.559$; ausgezeichnet: $N=24, A M=2.77, s=.968$; solide: $N=25, A M=2,67, s=.820$ ) 
Betrachtet man die Noten-Teilstichproben mit aussagekräftigem $\mathrm{N}$ in Abbildung 5, dann stellt man fest, dass sich solide und ausgezeichnete Kandidatinnen und Kandidaten in ihrer geäusserten Theoriedistanz kaum unterscheiden, hingegen ist der tiefere Wert der Studierenden, die mit einer exzellenten Note abgeschossen haben, offensichtlich. Die Note setzt sich aus einem gewichteten Mittel zusammen, das aufgrund der separaten Beurteilung einer schriftlichen Unterrichtsvorbereitung, der Unterrichtsdurchführung und eines anschliessenden Kolloquiums zustande kommt. Eine nachträgliche Überprüfung der drei unterschiedlich gewichteten Einzelkomponenten hat ergeben, dass die schriftliche Unterrichtsvorbereitung unabhängig zur Theoriedistanz variiert. Bedeutsam waren die Performanzvariablen «Durchführung» und «Kolloquium».

\section{Diskussion}

In diesem Beitrag wurde Komponenten des Interesses von Lehramtsstudierenden nachgegangen, die im Modell der reflexiven Praxis, insbesondere für die Verbindung zwischen Theorie und Praxis als bedeutsam gelten können.

Diagnostische Resultate: Im Einklang mit den theoretischen Vorüberlegungen gelang es, drei für die reflexive berufliche Entwicklung als bedeutsam erachtete Interesseskalen empirisch zu bestätigen. Insgesamt hat sich herausgestellt, dass auf Seiten der Lernenden eine überaus grosse Bereitschaft zur berufsbezogenen Reflexion vorausgesetzt werden kann. Pragmatismus und Theoriedistanz streuen hingegen um die theoretische Skalenmitte.

Bedingungsfaktoren: Inwieweit sich aus dem diagnostizierten Interesse auch eine intensivere Verknüpfung zwischen Theorie und Praxis einstellt, kann durch strukturelle Bedingungen in der Ausbildung mehr oder weniger unterstützt werden. Vor allem Theoriedistanz ist eine Grösse, die mit den Gegebenheiten der Ausbildungskonzeption relativ deutlich variiert. In dieser Hinsicht hat sich eine erweiterte standardorientierte Ausbildung als vorteilhaft erwiesen. Ein auf den Unterricht abgestimmtes Theoriecurriculum, der Wechsel zwischen theoretischen und praktischen Erfahrungen, verbunden mit entsprechenden Dokumentationsleistungen, scheinen ihre Wirkung nicht zu verfehlen (vgl. Niggli, 2001). Im Rahmen der Standardorientierung ist allerdings eine Übersetzungsleistung zu erbringen, und zwar aus den Spezialdiskurs der Fachdisziplin in die allgemeine Sprache der Praxis (Baeriswyl \& Niggli, 2003; Niggli, 2000). Dies zeigt der Rükkgang der Theoriedistanz im letzten Semester in der Ausbildung der Standardgruppe, wo in erheblichem Masse mit adaptierten theoretischen Informationen gearbeitet wird. Ähnliche Effekte konnten in einer früheren Untersuchung von Nölle (2002) beobachtet werden. Die Autorin hatte dieselbe Ausbildungsinstitution bereits zu einem früheren Zeitpunkt mit anderen Ausbildungsgängen ver- 
glichen. In wesentlich geringerem Masse variiert das Interesse an der Reflexion mit der Ausbildungsinstitution. Zusätzlich scheint das Geschlecht einen gewissen Einfluss zu haben. Angesichts der generell hohen Werte der Reflexion sind diese Differenzen weniger ausschlaggebend. Demgegenüber scheint der Pragmatismus der Studierenden eine sehr viel konstantere Grösse zu sein. Jedenfalls wird er von den vier untersuchten Bedingungsvariablen nicht beeinflusst. Die Veränderung pragmatischer Grundinteressen ist möglicherweise nur innerhalb eines relativ langen Zeitraumes zu erwarten. Vermutlich sind sie abhängig von praktischen Erfahrungen und Vorbildern und durch die Ausbildungsinstitution nur unwesentlich zu beeinflussen. Der epistemologischen Differenz zu theoretischen Aussagen entspricht es im Übrigen durchaus, dass pragmatische Ausrichtungen nicht zwingend Abwertung von Theorie zur Folge haben. Vereinbar ist diese Sichtweise ebenfalls mit dem Modell der Reflexion von Griffiths \& Tann (1992). Beide Systeme können sich zwar indirekt aufeinander beziehen, aber nicht füreinander Entscheidungen treffen. Diese Annahme wird im Übrigen durch die geringe Korrelation der beiden Interesseskalen gestützt.

Auswirkungen der Theoriedistanz: Die Verwissenschaftlichung des Könnens (Wissen FÜR die Praxis), die in einer Ausbildung erreicht werden soll, ist nur dann ein realistisches Ziel, wenn das Interesse an wissenschaftlichen Aussagesystemen mit besseren didaktischen Leistungen verbunden ist. Als Bedingungsgrösse für didaktisches Können hat sich die Theoriedistanz empirisch bestätigen lassen. Studierende mit exzellenten Leistungen in der praktischen Abschlussprüfung äusserten insgesamt eine deutlich niedrigere Theoriedistanz als die übrigen Kandidatinnen und Kandidaten. Auch wenn damit die Relevanz des wissenschaftlichen Wissens für die beurteilten Kompetenzen nicht im Einzelnen geklärt ist, besteht dennoch ein Indiz für die Betonung wissenschaftlicher Aussagensysteme als konstitutivem Element der Profession. Dazu wäre es wünschenswert, die Zusammenhänge längsschnittlich an neuen Stichproben und in unterschiedlichen Ausbildungsinstitutionen empirisch zusätzlich zu differenzieren. Die vorliegende querschnittliche Untersuchung hat eher Pilotcharakter und beschränkt sich auf eine einzelne Ausbildungsinstitution. Damit sind die Resultate auch abhängig von der in dieser Institution etablierten Beurteilungspraxis, beispielsweise der Anzahl der als hervorragend beurteilten Leistungen.

Konsequenzen für die Ausbildung: Für Ausbildungsinstitutionen lassen sich aus den vorliegenden Daten vorläufig folgende Überlegungen anstellen. Von den genannten drei Interessefaktoren hat sich die Theoriedistanz als Schlüsselvariable erwiesen. Ihr wäre somit Beachtung zu schenken. Eine erhebliche Distanz gegenüber wissenschaftlichen Aussagesystemen ist möglicherweise dann zu erwarten, wenn man befürchtet, durch die Reflexion bestehendes Handlungswissen bzw. die entsprechende Verhaltenssicherheit zu gefährden. Dies könnte dann der Fall sein, wenn es der Ausbildung nicht gelingt, Theorie und Praxis ein- 
leuchtend aufeinander zu beziehen. Informationen über das Ausmass der vorhandenen Theoriedistanz könnten für bestimmte Ausbildungsgänge deshalb ein relevantes Feedback sein. Eine Abwertung pragmatischer Erfahrungen der Studierenden als «unreflektiert» ist aufgrund der vorliegenden empirischen Daten im Weiteren nicht angezeigt. Dies entspräche im Übrigen auch nicht den getroffenen theoretischen Vorannahmen. Erfolgversprechender dürfte vielmehr die Spiegelung pragmatischer Grundhaltungen im Rahmen praxistauglicher wissenschaftlicher Theoriesysteme sein. Denn im «Handeln nur lernt man die Kunst, erlangt man Tact, Fertigkeit, Gewandtheit, Geschicklichkeit: aber selbst im Handeln lernt die Kunst nur der, welcher vorher im Denken die Wissenschaft gelernt, sie sich zu eigen gemacht, sich durch sie gestimmt, - und die künftigen Eindrücke, welche die Erfahrung auf ihn machen sollte, vorbestimmt hatte» (Herbart, 1964, S. 286f.).

\section{Literatur}

Baeriwyl, F. \& Niggli, A. (2003). Skript zur Allgemeinen Didaktik. Freiburg: Departement Erziehungswissenschaften der Universität. Abteilungen Ausbildung LDS I und LDS II.

Berliner, D.C. (1992). The nature of expertise in teaching. In F. Oser, A. Dick \& J.-L. Patry, (Hrsg.), Effective und responsible teaching. The new synthesis (S. 227-248). San Francisco: Jossey-Bass.

Carter, K. (1990). Teachers knowledge and learning to teach. In W.R. Houston, M. Habermann \& M. Sikula (Hrsg.), Handbook of research in teacher education (S. 291-310). New York: Macmillan.

Cochran-Smith, M. (2001). Constructing outcomes in teacher education: Policy, practice and pitfalls. In Education Policy Analysis Archives 9. Stand 13.7.2001. [Html-Dokument: www.epaa.asu.edulepaalv9n11.html].

Dewe, B. \& Radtke, F.-O. (1991). Was wissen Pädagogen über ihr Können? Professionstheoretische Überlegungen zum Theorie-Praxis-Problem in der Pädagogik. In J. Oelkers \& H.E. Tenorth, (Hrsg.). Pädagogisches Wissen, 27. Beiheft der Zeitschrift für Pädagogik (S. 143 -162). Weinheim: Beltz.

Dewe, B., Ferchhoff, W. \& Radtke, F.-O. (1992). Das «Professionswissen» von Pädagogen. Ein wissenschaftstheoretischer Rekonstruktionsversuch. In dies. (Hrsg.) Erziehen als Profession. Zur Logik professionellen Handelns in pädagogischen Feldern (S. 70-91). Opladen: Leske + Budrich.

Dick, A. (1994). Vom unterrichtlichen Wissen zur Praxisreflexion. Bad Heilbrunn: Klinkhardt.

Griffiths, M. \& Tann, S. (1992). Using reflective practice to link personal and public theories. Journal of Education for Teaching, 18, (1), 69-84.

Groeben, N. (1986). Handeln, Tun, Verhalten als Einheiten einer verstehend-erklärenden Psychologie. Tübingen: Francke.

Hatton, N. \& Smith, D. (1995). Reflection in teacher education: towards definition and implementation. Teaching and Teacher Education, 11, (1), 33-49.

Havers, N. \& Toepell, S. (2002). Trainingsverfahren für die Lehrerausbildung im deutschen Sprachraum. Zeitschrift für Pädagogik, 48, (2), 174-193.

Herbart, J.F. (1964). Zwei Vorlesungen über Pädagogik. In K. Kehrbach \& O. Flügel (Hrsg.), Sämtliche Werke, Bd. 1 (S. 279-290). Aalen: Scientia. 
Herzog, W. (1995). Reflexive Praktika in der Lehrerinnen- und Lehrerbildung. Beiträge zur Lehrerbildung, 13, (3), 253-273.

Koch-Prieve, B. (1997). Grundlegung einer Didaktik der Lehrerbildung. Der Beitrag der wissenspsychologischen Professionsforschung und der humanistischen Pädagogik. In M. Bayer, U. Carle \& J. Wildt (Hrsg.) Brennpunkt Lehrerbildung. Strukturwandel und Innovationen im europäischen Kontext (S. 139-163). Opladen: Leske + Budrich.

Koch-Prieve, B. (2002). Grundlagenforschung in der Lehrerinnenbildung. Zeitschrift für Pädagoagik, 48, (1), 1-9.

Krapp, A. (1998). Entwicklung und Förderung von Interessen im Unterricht. Psychologie in Erziehung und Unterricht, 45, (3), 185-201.

Mandl H. \& Gerstenmaier, J. (2000). Die Kluft zwischen Wissen und Handeln. Göttingen: Hogrefe.

Messner, H. \& Reusser, K. (2000). Berufliches Lernen als lebenslanger Prozess. Beiträge zur Lehrerbildung, 18, (3), 277-294.

Munby, H., Russel, T. \& Martin, A. (2002). Teachers knowledge and how it develops. In V. Richardson. Handbook of Research on Teaching (S. 877-904). Washington DC: AERA.

Niggli, A. (2000). Lernarrangements erfolgreich planen. Didaktische Anregungen zur Gestaltung offener Unterrichtsformen. Aarau: Sauerländer.

Niggli, A. (2001). Portfolios und der Theorie-Praxis-Bezug mit Ausbildungsstandards. Journal für LehrerInnenbildung, 1, (4), 26-33.

Nölle, K. (2002). Probleme der Form und des Erwerbs unterrichtsrelevanten pädagogischen Wissens. Zeitschrift für Pädagogik, 48, (1), 48-67.

Oser, F. (1997). Standards in der Lehrerbildung. Beiträge zur Lehrerbildung, 15, (1), 26-37.

Schaefers, Ch. \& Koch, S. (2000). Neue Veröffentlichungen zur Lehrerforschung. Zeitschrift für Pädagogik, 46, (4), 601-624.

Schiefele, U., Krapp, A., Wild, K.P. \& Winteler, A. (1993). Der Fragebogen zum Studieninteresse (FSI). Diagnostica, 39, (4), 335-351.

Schön, D.A. (1983). The reflective practitioner. London: Temple Smith.

Schön, D.A. (1987). Educating the reflective practitioner: Toward a new design for teaching and learning in the professions. San Francisco: Jossey-Bass.

Schön, D.A. (1991). The reflective turn. Case studies in and on educational practice. New York: Teachers College Press.

Staub, F. (2001). Fachspezifisch-pädagogisches Coaching: Theoriebezogene Unterrichtsentwicklung zur Förderung von Unterrichtsexpertise. Beiträge zur Lehrerbildung, 19, (2), 175-198.

Terhart, E., Czerwenka, K., Ehrich, K., Jordan, F. \& Schmidt, H. J. (1994). Berufsbiographien von Lehrern und Lehrerinnen. Frankfurt: Lang.

Terhart, E. (2002). Nach Pisa. Hamburg: Europäische Verlagsanstalt.

Wahl, D. (1991). Handeln unter Druck. Der weite Weg vom Wissen zum Handeln. Beiträge zur Lehrerbildung 9, (2), 157-174.

Wahl, D. (2002). Mit Training vom trägen Wissen zum kompetenten Handeln? Zeitschrift für Pädagogik, 48, (2), 227-241.

Wildt, J. (1996). Reflexive Lernprozesse. In D. Hänsel, L. Huber, (Hrsg.) Lehrerbildung neu denken und gestalten (S. 91-107). Weinheim: Beltz.

West, L. \& Staub, F. (2003). Content-focused coaching. Transforming mathematics lessons. Portsmouth: Heinemann. Pittsburgh PH: University of Pittsburgh.

Zeichner, K.M. \& Liston, D.P. (1996). Reflective teaching: An introduction. Mahwah, New Jersey: Lawrence Erlbaum Associates, Publishers.

Schlagworte: Fragebogen, Hochschule, Lehrerbildung 


\section{Quelles composantes d'une réflexion sur la pratique professionnelle intéressent les enseignants débutants et les futurs enseignants? - Structure factorielle d'un questionnaire et premiers résultats empiriques}

\section{Résumé}

Cette contribution présente le développement d'un questionnaire qui mesure les différents intérêts que des étudiants de DES (diplôme d'enseignement secondaire) accordent à la réflexion sur la pratique professionnelle. Le processus prend en considération trois facteurs : (a) l'intérêt pour la réflexion, (b) la distance à la théorie et (c) le pragmatisme. Après quelques études pilotes, le questionnaire a été testé sur un échantillon (A) de 210 étudiants. Les résultats empiriques confirment la structure des facteurs postulée dans la théorie. Dans une enquête consécutive, elle-même vérifiée par un test effectué sur un échantillon (B) de 79 personnes, on peut constater que tant l'intérêt des étudiants pour la réflexion que leur distance vis-à-vis de la théorie se développent de manière différente selon les établissements de formation. Les étudiants qui obtiennent la meilleure note à l'examen pratique montrent, de manière significative, une distance moindre à la théorie. Ces résultats confirment l'importance du concept.

Mots clés: formation des enseignants, université, questionnaire

\section{Quali componenti dello sviluppo riflessivo della professione interessano gli insegnanti in formazione? Struttura di un questionario e primi risultati empirici}

\section{Riassunto}

Il contributo presenta lo sviluppo di un questionario per il rilevamento dei tipi d'interesse dei docenti in formazione relativamente alla riflessione della loro pratica professionale. L'approccio considera tre fattori: a) l'interesse per la riflessione, b) la distanza rispetto alla teoria e c) il pragmatismo. A seguito di diversi studi pilota il questionario è stato verificato su un campione (A) di 210 studenti. I risultati empirici hanno confermato la struttura fattoriale ipotizzata. Una seconda inchiesta su un campione (B) di 79 persone ha mostrato che l'interesse per la riflessione e la distanza rispetto alla teoria si sviluppano differentemente a seconda dell'istituzione formativa. Inoltre gli studenti con le valutazioni migliori nell'esame di pratica manifestano una distanza rispetto alla teoria significativamente minore. I risultati confermano la rilevanza del concetto.

Parole chiave: formazione degli insegnanti, questionario, università 


\section{Which elements of reflection about professional practice interest future teachers ? - Factors structure of a questionnaire and first empirical results}

\section{Summary}

The following article reports about the construction of a questionnaire which measures the patterns of interest in professional reflection during their teacher training. Three conceptual factors are relevant: (a) interest in reflection (IR), (b) distance from theory (DT) and (c) pragmatism (P). Following several pilot studies, the questionnaire was tested in a random sample of 210 students. The empirical findings confirmed the theoretically postulated factor structure. A followup study (N=79), showed that interest in reflection (IR) as well as distance from theory (DT) developed differently depending on the teacher training institution. Additionally, students with the highest marks in professional practice exams had significantly less distance from theory. The results confirm the relevance of the concepts.

Key words: questionnaire, teacher education, university 